\title{
Phase transition and heterogeneous/epitaxial nucleation of hydrated and anhydrous theophylline crystals
}

\author{
Naír Rodríguez-Hornedo ${ }^{a}$, David Lechuga-Ballesteros ${ }^{a}$ and Hsiu-Jean $\mathrm{Wu}^{\mathrm{b}}$ \\ a College of Pharmacy, The University of Michigan, Ann Arbor, MI 48109-1065 (USA) and b College of Pharmacy, \\ The University of Arizonu, Tucson, AZ 85721 (USA)
}

(Received 9 September 1991)

(Modified version received 12 February 1992)

(Accepted 9 March 1992)

Key words: Phase transformation; Epitaxy; Crystallization; Dissolution; Anhydrous theophylline; Theophylline monohydrate

\section{Summary}

Theophylline crystallizes in the anhydrous or monohydrate form. The solubility of the anhydrous form exceeds the solubility of the monohydrate form below $60^{\circ} \mathrm{C}$. The anhydrous: hydrate phase transition of theophylline in buffered suspensions has been studied by measurement of the supersaturation profile and the crystal size distributions. The role of crystallographic parameters in the nucleation and growth of monohydrate crystals, during dissolution of the anhydrous phase, has been investigated. A solvent-mediated route has been identified and can be described in terms of the dissolution kinetics of the anhydrous phase and the nucleation and growth kinetics of the hydrated phase. The growth rate of the monohydrate crystals depends on the square of the supersaturation and is independent of the stirring rate, which suggests a surface-controlled growth process. The dissolution rate of the anhydrous crystals depends on the 1.5 order of the undersaturation and is inversely proportional to the square root of the crystal size. Anhydrous theophylline crystals act as heterogeneous nucleation substrates for the crystals of the monohydrate phase and these grow epitaxially on the anhydrous crystals. Based on the kinetic equations for dissolution of the anhydrous form and crystallization of the monohydrate form, a computer simulation has been developed that predicts the changes in the concentration of theophylline in solution during the phase transition.

\section{Introduction}

The presence of a metastable form during processing or in the final dosage form often leads to instability of drug release as a result of a phase transformation. Recent studies show erratic dis-

Correspondence to: N. Rodríguez-Hornedo, College of Pharmacy, The University of Michigan, Ann Arbor, MI 48109-1065, U.S.A. solution from anhydrous theophylline tablets stored at high relative humidities (Ando et al., 1986; Herman et al., 1989) or prepared by a wet granulation process (Herman et al., 1988). These have been explained by a phase transformation from the anhydrous to the monohydrate form. Theophylline is a widely used drug for the treatment of asthma. It has a $\mathrm{p} K_{\mathrm{a}}$ of 8.7 (Windholz, 1983) and the solubility of the anhydrous form exceeds that of the monohydrate form, below $60^{\circ} \mathrm{C}$. 
Anhydrous to hydrate phase transformations may occur by either of two processes, when the solid is in contact with water: (i) a solid-solid transformation in which water molecules are incorporated into the crystal lattice while remaining in the solid state or (ii) a solvent-mediated process where the anhydrous solid dissolves, and creates the necessary supersaturation for the nucleation and growth of monohydrate crystals.

It has been observed that during dissolution of anhydrous theophylline from a disk (De Smidt et al., 1986) the monohydrate form crystallizes on the surface of the anhydrous form and that the transformation kinetics depends on the boundary layer thickness. Such observations suggest a solvent-mediated phase transformation. A critical question is whether heterogeneous nucleation and even epitaxy facilitate the birth and growth of crystals of the stable phase - if the presence of a metastable or unstable solid phase promotes nucleation, since spontaneous or homogeneous nucleation has not been observed. The surface of the anhydrous phase may act as a nucleation substrate for the monohydrate form by either decreasing the solution-nucleus interfacial cnergy or by a crystal lattice match. The latter would result in oriented, epitaxial, growth of the monohydrate crystals on the anhydrous phase. The higher the affinity of the solute for the solid substrate, the smaller the energy required to form the nucleus, and nucleation may occur at very low supersaturations (Walton, 1969). Thus, the theophylline concentration in solution may never reach the homogeneous nucleation levels.

Previous studies (Wadke and Reier, 1972; Fokkens et al., 1983; De Smidt et al., 1986) emphasize the consequences of a transformation, due to the crystallization of theophylline monohydrate, on the dissolution kinetics of anhydrous theophylline. However, the mechanisms and kinetics by which theophylline monohydrate crystals nucleate and grow are not understood. The objective of this study was to elucidate the kinetics of the anhydrous to hydrate transformation of theophylline in a buffered suspension by taking into consideration the dissolution of the anhydrous phase and the nucleation and growth of the monohydrate phase. The dissolution kinetics of anhydrous theophylline crystals and growth kinetics of monohydrate crystals have been reported (Wu, 1990; Rodríguez and $W u, 1991$ ). In order to gain insight about the transformation mechanism, the concentration of theophylline in solution was monitored, and the evolution of the crystal size distribution (CSD) was measured. Furthermore, to investigate the importance of crystallographic parameters - the possibility of heterogeneous nucleation of monohydrate crystals and epitaxial growth on the anhydrous form - the transformation was studied by microscopic observation.

\section{Materials and Methods}

\section{Materials}

Anhydrous theophylline was obtained from Sigma Chemical Co. (St. Louis, MO), dried for 24 $h$ in an oven at $100^{\circ} \mathrm{C}$ and stored in a desiccator oyer calcium sulfate at room temperature. Large single crystals of anhydrous theophylline were grown from chloroform by slow evaporation, for microscopic studies. Theophylline monohydrate crystals were prepared by crystallization from phosphate buffer ( $\mathrm{pH}$ 6), that was supersaturated by dissolving the anhydrous form. Monohydrate crystals were dried at room temperature and stored over a saturated solution of sodium bromide (relative humidity $60 \%$ ). The water used in this study was filtered through a double-deionized purification system (Milli $Q$ Water System from Millipore Co.). Phosphate buffer ( $\mathrm{pH} \mathrm{6)}$ was prepared from $0.067 \mathrm{M}$ potassium phosphate monobasic solution and $0.067 \mathrm{M}$ sodium phosphate dibasic solution. The ionic strength of the buffer was adjusted to $0.15 \mathrm{M}$ with potassium chloride. These reagents were purchased from Aldrich Chemical Co. All solutions were filtered through a $0.45 \mu \mathrm{m}$ filter prior to use.

\section{Assay and identification}

Theophylline concentration in solution was measured by UV spectrophotometry with a Beckman DU-8 spectrophotometer at $270 \mathrm{~nm}$. An IR spectrophotometer (Beckman IR-33) was used to determine the IR spectra of anhydrous and monohydrate theophylline crystals. Differential 
scanning calorimetry (DSC) was performed with a Dupont 1090 Thermal Analyzer.

\section{Solubility}

The solubility of theophylline monohydrate was measured over the temperature range $8-40^{\circ} \mathrm{C}$, in $0.067 \mathrm{M}$ phosphate buffer $(\mathrm{pH} 6)$ of ionic strength $0.15 \mathrm{M}$. Equilibrium was achieved by dissolution and crystallization.

The solubility of the anhydrous form was calculated from the initial dissolution rate of anhydrous theophylline from a disk (Shefter and Higuchi, 1963; Wadke and Reier, 1972). Anhydrous or monohydrate crystals were compressed at a pressure of $3000 \mathrm{psi}\left(\mathrm{lb} / \mathrm{inch}^{2}\right)$. The die was then placed in a holder and lowered into the dissolution medium ( $400 \mathrm{ml}$ of $\mathrm{pH} 6$ buffer) that had been equilibrated at the desired temperature. The solution was stirred with a magnetic stirrer and aliquots were taken at various times for analysis.

\section{Theophylline monohydrate seeds}

A saturated solution of theophylline monohydrate in $\mathrm{pH} 6$ phosphate buffer at $25^{\circ} \mathrm{C}$ was chilled in an ice bath $\left(-10^{\circ} \mathrm{C}\right)$ to $0^{\circ} \mathrm{C}$ with constant stirring. The suspension was filtered after the crystal seeds reached the desired size. The seeds were resuspended and aged in saturated buffer for a period of 2 weeks at room temperature. The seeds were identified by IR spectroscopy and the number per $\mathrm{ml}$ and CSD measured. The seed suspension contained $76 \mathrm{mg}$ monohydrate crystals per $\mathrm{ml}$.

\section{Anhydrous to hydrate transformation}

Experiments were carried out with a suspension of anhydrous theophylline $(250 \mathrm{ml})$ at $10^{\circ} \mathrm{C}$ in a $300 \mathrm{ml}$ jacketed beaker at a stirring rate of $750 \mathrm{rpm}$. The initial concentration of the solution was adjusted to the solubility of the anhydrous form $(8.75 \mathrm{mg} / \mathrm{ml})$ prior to the addition of $5.5 \mathrm{~g}$ of anhydrous crystals. After a few seconds a suspension of monohydrate seed crystals was added. 2 -ml aliquots were taken, and the theophyllinc concentration in solution measured before and during the transformation.
The concentration profiles were measured during the transformation and the effect of seeding with monohydrate theophylline crystals was investigated. The transformation was carried out at monohydrate seed levels of $0 \%, 0.5 \%(38 \mathrm{mg})$ and $2.0 \%(152 \mathrm{mg})$. The concentration profile was also measured in the absence of anhydrous theophylline at a monohydrate seed level of $0.5 \%$. The amount of monohydrate seeds is expressed as the percent weight of monohydrate theophylline crystals precipitated at the end of the process.

The CSD was monitored as a function of time with an Elzone 180XY particle counter (Particle Data Labs, Elmhurst, IL). Crystal sizes (volume equivalent size) within the range $12-120 \mu \mathrm{m}$ were measured by using a $300 \mu \mathrm{m}$ orifice size. After the transformation was completed the crystals were identified by IR spectroscopy to be the monohydrate form.

In a separate series of experiments the dissolution of anhydrous theophylline and the growth of monohydrate crystals were studied. The dissolution of anhydrous theophylline (50 mg) in $\mathrm{pH} 6$ phosphate buffer $(200 \mathrm{ml})$ was evaluated at various undersaturations by monitoring the theophylline concentration and the CSD (Wu, 1989). A similar procedure was followed to study the crystallization kinetics of the monohydrate phase, and the growth rate dependence on supersaturation (Rodríguez and $\mathrm{Wu}, 1991$ ). The driving force for dissolution of the anhydrous phase is

$\sigma_{\mathrm{A}}=\frac{S_{\mathrm{A}}-C}{S_{\mathrm{A}}}$

where $S_{\mathrm{A}}$ is the solubility of anhydrous theophylline crystals, and $C$ denotes the theophylline concentration in solution. The driving force for growth of the monohydrate crystals is

$\sigma_{\mathrm{H}}=\frac{C-S_{\mathrm{H}}}{S_{\mathrm{H}}}$

where $S_{\mathbf{H}}$ is the solubility of monohydrate theophylline crystals. 


\section{Microscopic studies}

Single crystals of anhydrous theophylline were added to a $\mathrm{pH} 6$ solution saturated with respect to the anhydrous form at room temperature $\left(25^{\circ} \mathrm{C}\right)$. Experiments were conducted in microtitration multi-well plates of $0.35 \mathrm{ml}$ capacity. The plate was placed on the stage of an inverted microscope (Nikon Diaphot) and the transformation was followed by direct observation. Similar experiments were performed with the commercially prepared theophylline of a polycrystalline nature (aggregated crystals).

\section{Results and Discussion}

\section{Solubility}

During dissolution of anhydrous theophylline crystals, the solution becomes supersaturated with respect to the hydrated form which precipitates and grows until the concentration reaches the solubility of the monohydrate modification. The maximum value observed may correspond to the solubility of the anhydrous form or could be the result of a steady-state, dissolution-crystallization process.

A concentration-time profile resulting from disk dissolution of anhydrous and monohydrate modifications is presented in Fig. 1. These results suggest a transition during the dissolution of the anhydrous form. The rate of increase of theophylline concentration in the dissolution medium is faster during the first $6 \mathrm{~min}$, then it becomes slower and parallels that of the monohydrate form. This indicates that during dissolution, the anhydrous theophylline undergoes a phase transition to the monohydrate form on the surface of the disk since there was no nucleation in the bulk of the solution. De Smidt and co-workers (1986) have confirmed the presence of theophylline monohydrate on the surface of anhydrous theophylline disks by IR spectroscopy and by microscopic observation.

This transition may be the result of a solid-solid or solvent-mediated transformation. In the former, the molecules rearrange themselves incorporating the water of hydration into the crystal lattice while remaining in the solid state, whereas

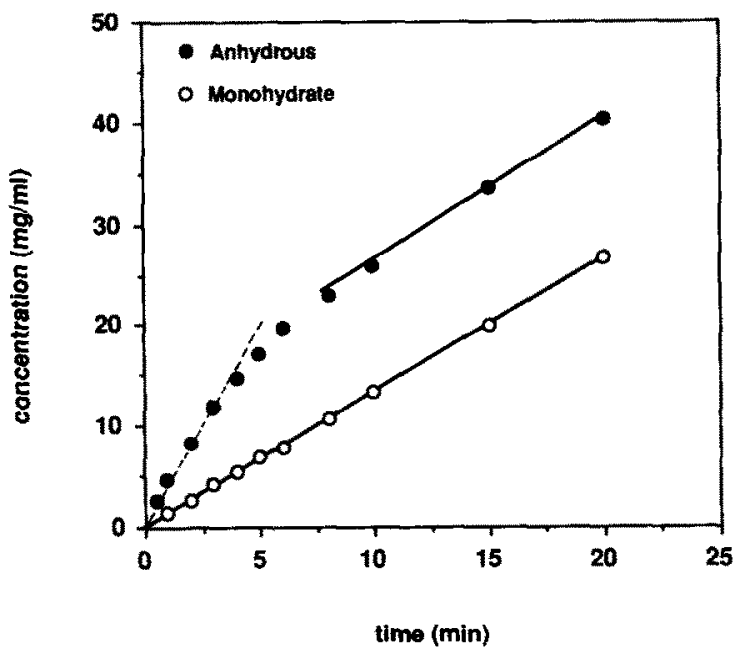

Fig. 1. Concentration profile during the dissolution of anhydrous and monohydrate theophylline from a disk at $10^{\circ} \mathrm{C}$. (-.....) Dissolution rate of anhydrous theophylline. ( Dissolution rate of monohydrate theophylline.

in the latter the anhydrous solid dissolves followed by crystallization of the monohydrate. Even though dissolution of the anhydrous (unstable) phase occurs in a solution where the bulk concentration of theophylline is below saturation with respect to the monohydrate (stable) phase, a solvent-mediated process is possible if the solvent adjacent to the disk surface is supersaturated.

The solubility of the anhydrous form was evaluated from the initial dissolution rate from a disk, while $C \ll S_{\mathrm{A}}$, according to (Shefter and Higuchi, 1963):

$S_{\mathrm{A}}=\frac{(\mathrm{d} C / \mathrm{d} t)_{0, \mathrm{~A}}}{(\mathrm{~d} C / \mathrm{d} t)_{0, \mathrm{H}}} S_{\mathrm{H}}$

This expression is valid because the dissolution rate constant is proportional to the diffusion coefficient, the boundary layer thickness and the surface area. Under constant hydrodynamic conditions and equal surface areas, both crystal forms should have the same value of the dissolution rate constant. The solubility curves for the anhydrous and monohydrate modification are presented in Fig. 2. 


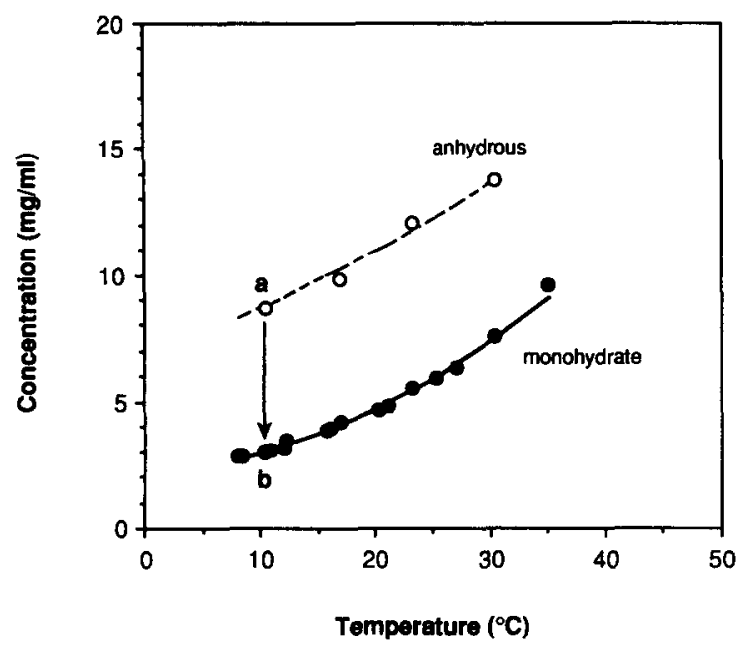

Fig. 2. Solubility dependence on temperature, for anhydrous and monohydrate theophylline in $\mathrm{pH} 6$ phosphate buffer. Points $a$ and $b$ represent the concentration range in which the phase transformation was studied.

\section{Phase transformation}

The anhydrous-monohydrate phase transition of theophylline was studied in a suspension at $10^{\circ} \mathrm{C}$. At this temperature the solubility of the anhydrous form is $8.75 \mathrm{mg} / \mathrm{ml}$ whereas that of the monohydrate has a value of $2.99 \mathrm{mg} / \mathrm{ml}$. Initially, anhydrous theophylline crystals were suspended in a solution of composition a (Fig. 2) which corresponds to the solubility of the anhydrous form. The transformation was completed at point $b$, when the solubility of the monohydrate form was reached.

Fig. 3 shows the CSDs measured during the transformation of $5.5 \mathrm{~g}$ of anhydrous theophylline and $0.5 \%$ monohydrate seeds. The amount of monohydrate seeds is expressed as $\%(\mathrm{w} / \mathrm{w})$ of the final mass of theophylline monohydrate precipitated. Several comments can be made about the distributions: (a) there is significant dissolution of the anhydrous crystals after $2 \mathrm{~min}$, as shown by the decrease in the number of large anhydrous crystals (plot $\mathrm{C}$ ); (b) growth of the monohydrate seeds is negligible in depleting the supersaturation and (c) a large number of monohydrate crystals nucleated and have grown to an average size of $25 \mu \mathrm{m}$, after $6 \mathrm{~min}$. These obser-
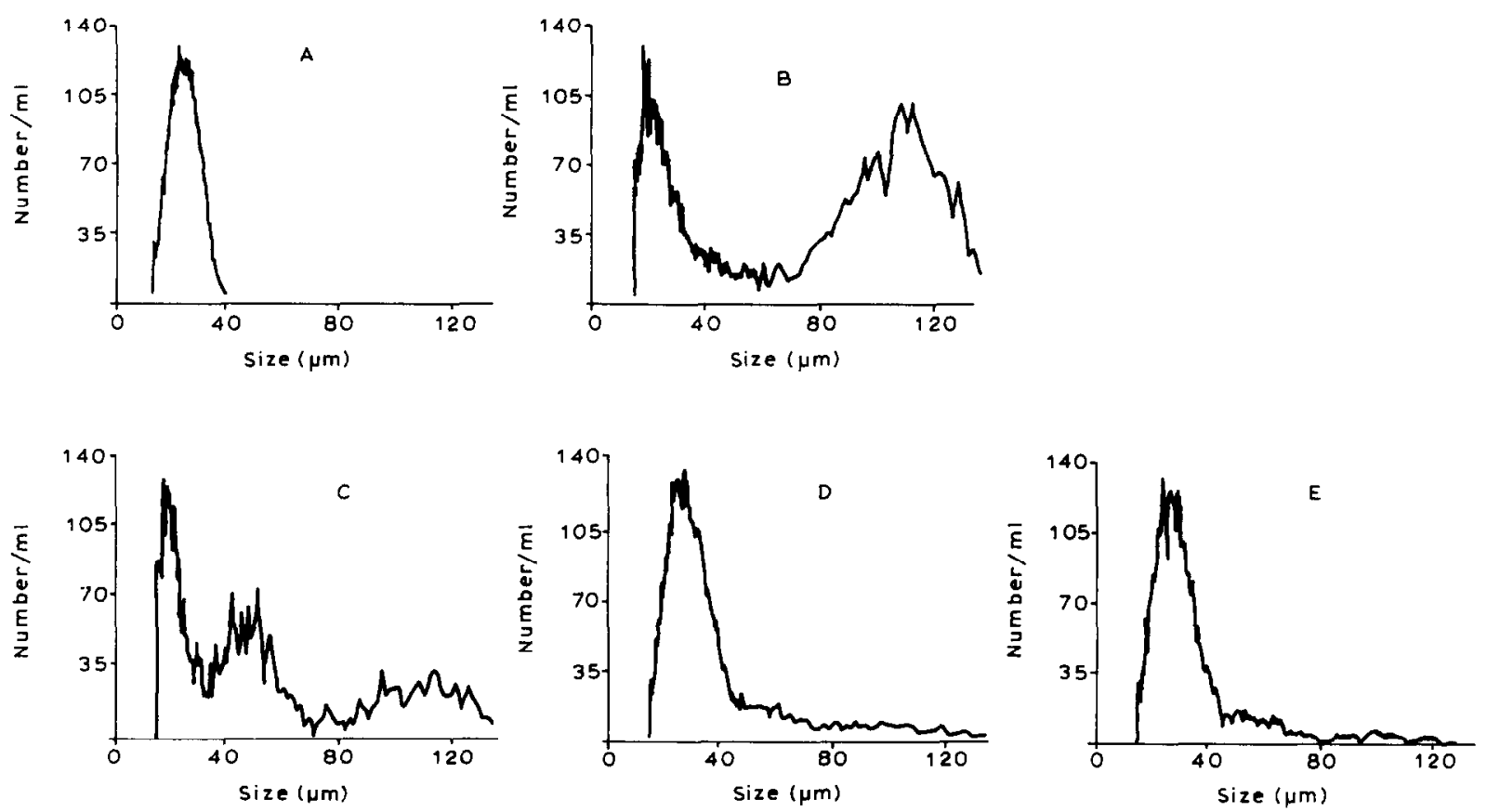

Fig. 3. CSD during the transformation of $5.5 \mathrm{~g}$ anhydrous theophylline in the presence of $0.5 \%$ monohydrate seeds. Initial distributions of monohydrate seeds (A) and anhydrous phase (B). Distributions during the transformation, at 2 min (C), 4 min (D) and $6 \mathrm{~min}(\mathrm{E})$. The samples were diluted $200 \times$ in panels $\mathrm{B}-\mathrm{E}$. 
vations confirm the process to be a solvent-mediated phase transformation rather than a solidstate hydration promoted by the solvent. If a solid-state mechanism were responsible for the transformation, the shape of the CSD would have been conserved.

The magnitude of the nucleation event is best seen by comparing the final number of theophylline monohydrate crystals as a function of the initial amount of anhydrous crystals, and the initial amount of monohydrate crystal seeds in Fig. 4. The final number of monohydrate crystals is strongly dependent on the amount of anhydrous phase present which indicates a heterogeneous nucleation mechanism. The presence of theophylline monohydrate crystals does not promote nucleation (secondary nucleation) since the final number of crystals is not dependent on the initial number of monohydrate crystal seeds.

Typical curves showing the variation in concentration as a function of time for solutions having initially the same amount of anhydrous theophylline crystals and supersaturation with respect to monohydrate, but different amounts of monohydrate seeds, are shown in Fig. 5. The curves usually consist of three stages. In the first

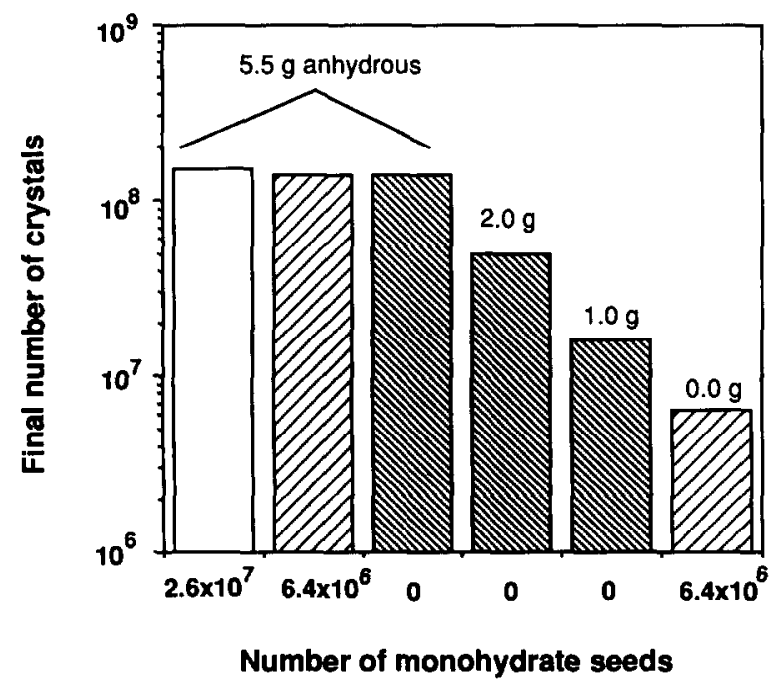

Fig. 4. Variation of the final number of hydrated theophylline crystals with the number of monohydrate seeds and mass of anhydrous phase added. A seed level of $0.5 \%$ corresponds to a number of crystals of $6.4 \times 10^{6}$ and $2.0 \%$ to $2.6 \times 10^{7}$.

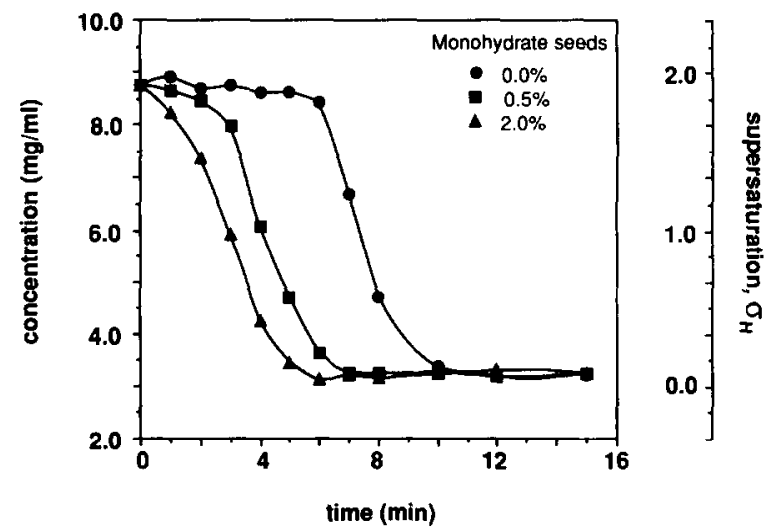

Fig. 5. Concentration profile during the transformation of 5.5 $\mathrm{g}$ of anhydrous theophylline in the presence of various levels of monohydrate seeds.

stage, the concentration remains constant for some time, so that the curves exhibit a plateau whose length depends on the initial amount of monohydrate seeds. Dissolution of anhydrous crystals is very slow because the solution is weakly undersaturated. Microscopic observation of the solid phase shows that this stage corresponds to the heterogeneous nucleation of monohydrate crystals. As the amount of monohydrate crystal seeds increased the length of the plateau decreased due to a larger surface area available for growth initially, whereas the final number of crystals remained constant. In the second stage, the theophylline concentration in solution decreases very rapidly, and the desupersaturation rate is independent of the number of monohydrate crystal seeds. This stage corresponds to nucleation and growth of theophylline monohydrate. Dissolution of the anhydrous form cannot compensate for the monohydrate crystallization. The supersaturation-time profile during monohydrate crystal growth in the absence of anhydrous phase is shown in Fig. 6. Nucleation is negligible and growth is mainly responsible for the decay in the supersaturation. In the last stage monohydrate crystals grow at a very low supersaturation and the concentration approaches the solubility of the monohydrate form. After describing the main stages of the anhydrous-hydrate transformation of theophylline in suspension, we consider in more detail the processes involved. 


\section{Anhydrous dissolution}

The dissolution rate of anhydrous theophylline crystals as a function of solution undersaturation under conditions similar to those encountered during the phase transformation experiments was measured. These experiments were carried out with a constant initial amount of anhydrous theophylline, at various degrees of undersaturation, $\sigma_{\mathrm{A}}$. To avoid corrccting for a change in shape factor during dissolution, only the initial dissolution rates were considered. The dissolution rate, $D$, was found to be size dependent, viz., the smaller crystals dissolved at a faster rate than the larger ones. The results are described by:

$D=k_{\mathrm{d}} \sigma_{\mathrm{A}}^{m} L^{b}$

where $k_{\mathrm{d}}$, the dissolution rate constant, has a value of 500 , the reaction order, $m$, has a value of $1.5, L$ represents the crystal volume diameter and the exponent $b$ has a value of -0.5 . The reaction order with respect to undersaturation for other systems (Amathieu and Boistelle, 1988) has been found to be in the range of one to two. A reaction order of one is taken to indicate that the rate-determining step is volume diffusion while for interfacial control the reaction order is two. The intermediate value obtained in this case may be due to the fact that heterogeneous nucleation on the anhydrous crystals disturbs the surface
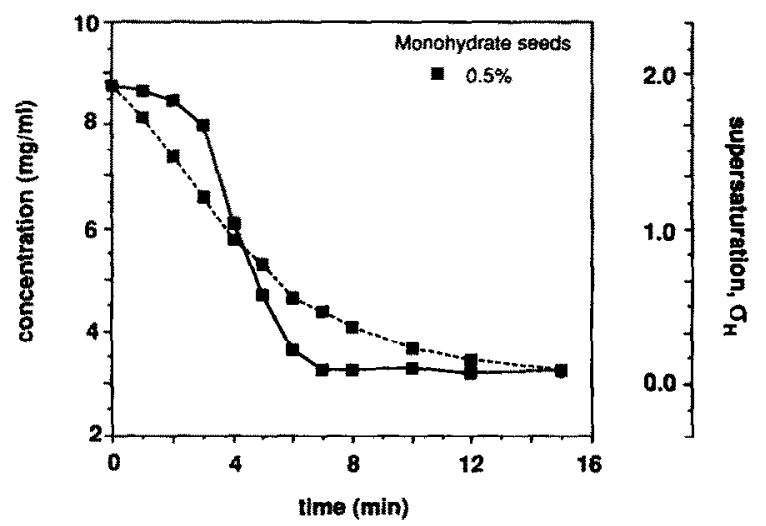

Fig. 6. Concentration profile during crystallization of theophylline monohydrate in the presence ( - ) and absence (.....) of anhydrous theophylline.

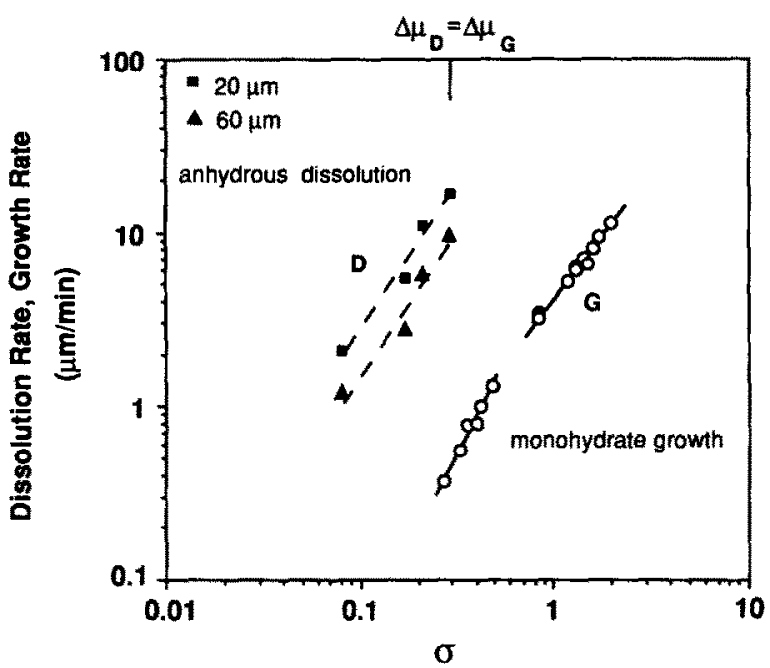

Fig. 7. Dependence of anhydrous theophylline dissolution rate and monohydrate growth rate on the driving force, $\sigma$, at $10^{\circ} \mathrm{C}$.

phenomena, since the solutions are supersaturated with respect to the monohydrate modification.

\section{Growth of monohydrate crystals}

The growth of theophylline monohydrate crystals has been studied as a function of the supersaturation (Rodríguez and Wu, 1991). In Fig. 7 the growth rate is plotted as a function of the supersaturation according to the linearized form of

$G=k_{\mathrm{g}} \sigma_{\mathrm{H}}^{n}$

where the growth rate constant, $k_{\mathrm{g}}$, has a value of 5.5 , and the growth exponent, $n$, has a value of 2.2 for supersaturations below 0.85 . This result is in agreement with the screw dislocation model. In this case, the growth rate can change linearly or parabolically with respect to the supersaturation (Burton et al,, 1951). At higher supersaturations, $n=1.5$ which may indicate a higher step density, where attachment of molecules to a step directly from the supersaturated phase is more probable than surface diffusion. It was also observed that the growth of theophylline monohydrate crystals is independent of the stirring rate and from the 
temperature dependence of the growth rate, the activation energy for crystal growth was determined to be $14.3 \mathrm{kcal} / \mathrm{mol}$.

Fig. 7 illustrates the influence of the driving force upon dissolution of anhydrous and crystallization of monohydrate theophylline. At the same departure from equilibrium, chemical potential, $\Delta \mu_{\mathrm{D}}=\Delta \mu_{\mathrm{G}}$ or $\sigma_{\mathrm{A}}=\sigma_{\mathrm{H}}$, dissolution proceeds at a faster rate than crystallization. The energy of activation for diffusion determined from the temperature dependence of the dissolution rate of theophylline from a disk was $4.4 \mathrm{kcal} / \mathrm{mol}$ (Wu, 1990). This confirms that growth of the monohydrate form is controlled by a surface reaction mechanism rather than by solute diffusion in the bulk. When dissolution data from a rotating disk are used to investigate a phase transformation, it is important to establish the rate-determining step. Interpretations can be misleading, since crystal growth has been frequently assumed to be diffusion-controlled.

\section{Heterogeneous nucleation and epitaxy}

To identify the crystallographic consequences on the heterogeneous nucleation mechanism, experiments have been carried out with single crystals and polycrystalline samples of anhydrous theophylline in contact with a solution saturated with respect to the anhydrous form. Through microscopic observation the following events were noticeable. The polycrystalline samples and single crystals of anhydrous theophylline were, within minutes, covered by crystallites of the hydrated phase as shown in Fig. 8a. The monohydrate crystals grew towards the solution while the anhydrous phase slowly dissolved (Fig. 8b). Towards

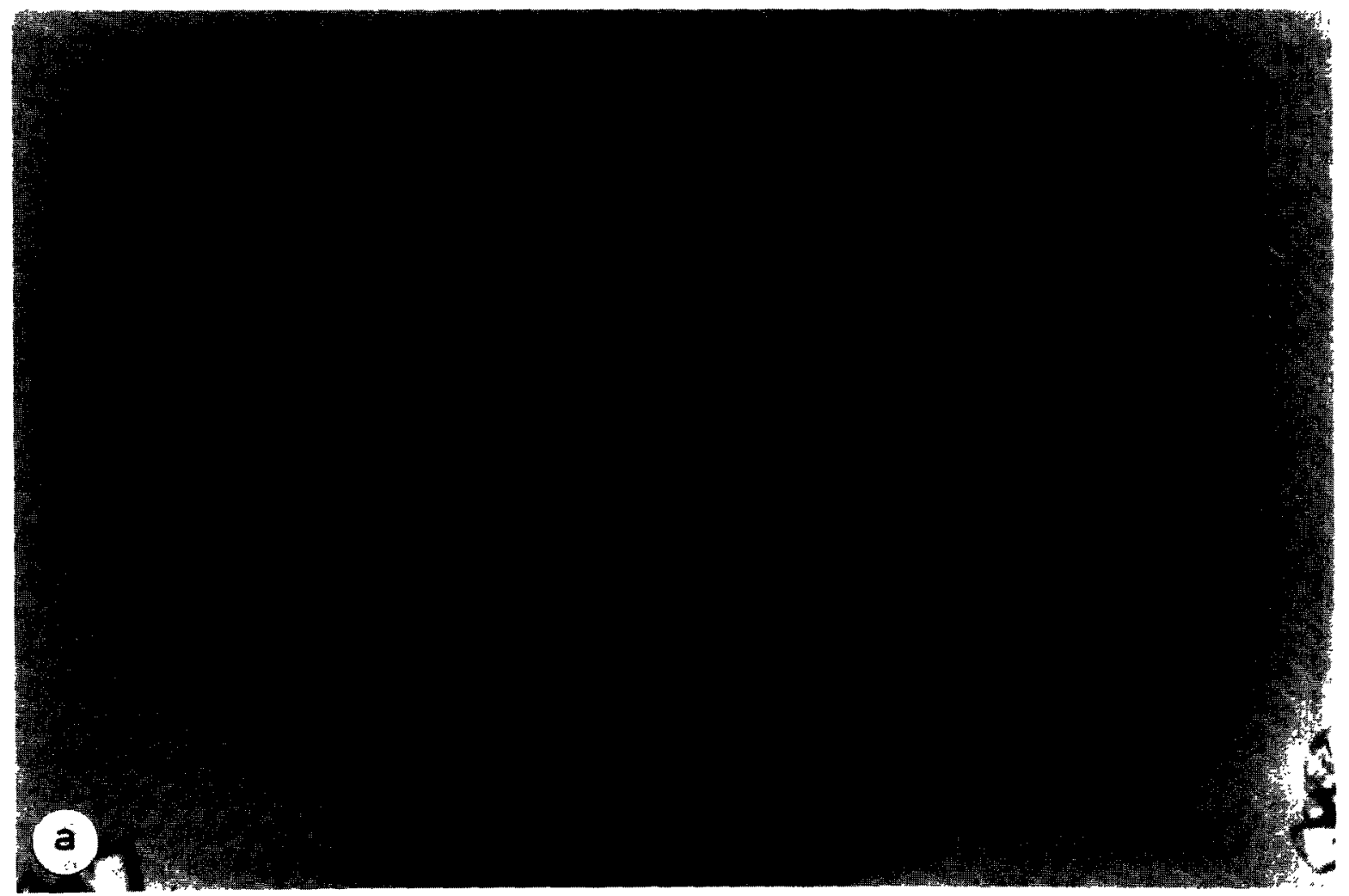

Fig. 8. Photographs displaying the heterogeneous nucleation of theophylline monohydrate on the surface of polycrystalline theophylline anhydrous, in contact with phosphate buffer saturated with respect to the anhydrous phase at $25^{\circ} \mathrm{C}$ : (a) 2 min and (b) 


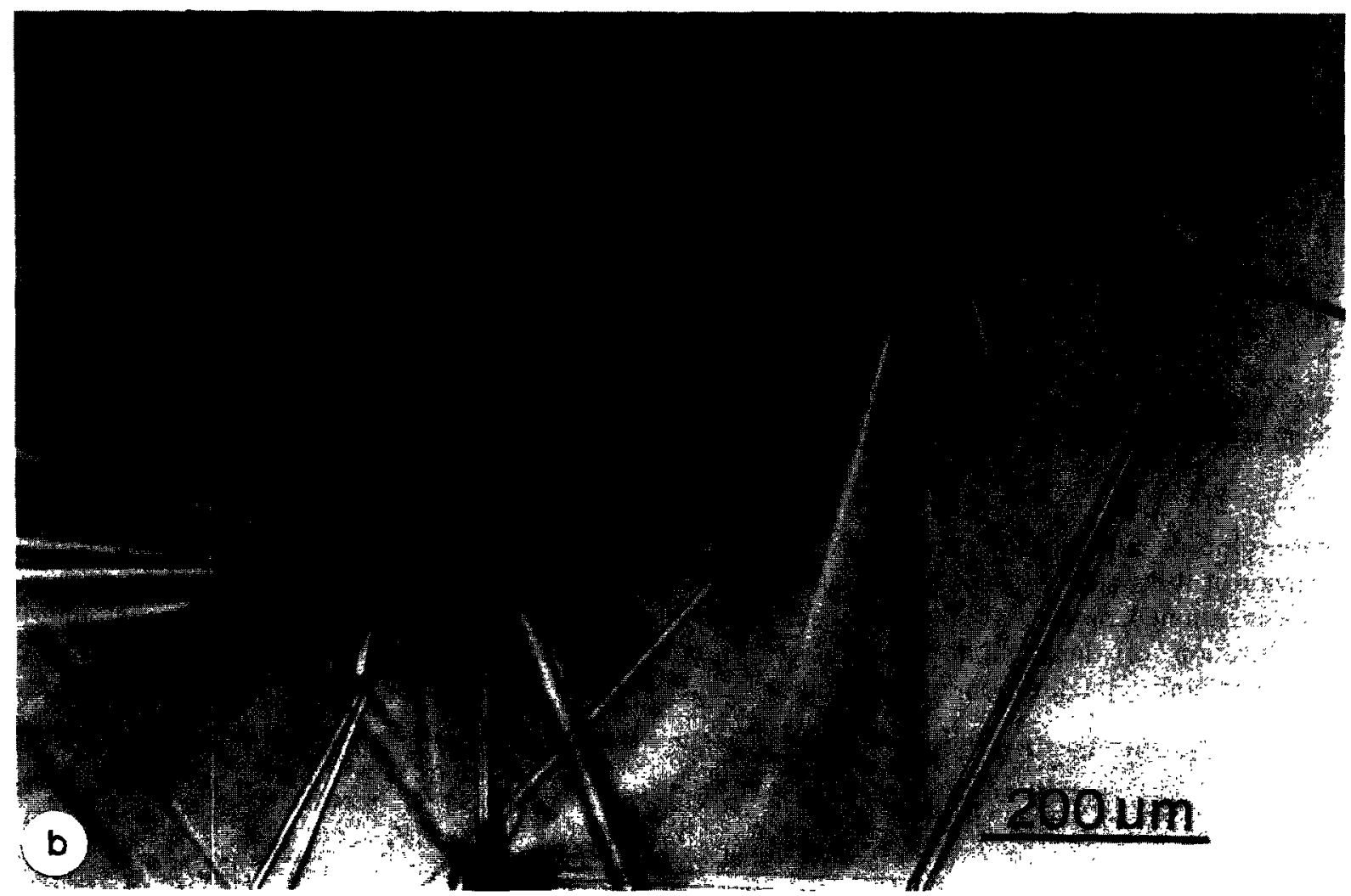

Fig. 8 b.

the end of the process traces of anhydrous crystals remained on the hydrated phase. Eventually $(24 \mathrm{~h})$ all the anhydrous phase dissolved. In the case of the polycrystalline sample, the hydrated crystals were randomly oriented on the anhydrous surface which hindered their dissolution. When anhydrous single crystals were used as the nucleating substrate, the monohydrate crystals grew aligned along the principal face of the anhydrous crystal as is illustrated in Fig. 9. The direction of fast growth of the monohydrate, $b$ crystallographic axis (Sutor, 1958), is superimposed on the direction of elongation of the anhydrous crystal, $b$ crystallographic axis. From the unit cell dimensions (Table 1), a lattice match along the $b$ axis is the most likely one. This confirms the epitaxial mechanism obscrved, as a result of a templatemediated nucleation. Fig. 9 portrays the crystallographic plate (010), which shows the molecular arrangement of theophylline monohydrate along its $b$ crystallographic axis.

Previous studies on the phase transitions of calcium sulfate hydrates (Amathieu and Boistelle, 1988), and hydrated and anhydrous uric acid (Boistelle and Rinaudo, 1981) have shown that heterogeneous nucleation occurs on the surface of the unstable phase. Each uric acid modification epitaxially grows on the other one and this has been explained by a crystallographic mechanism.

Simulation of the concentration profile during the phase transformation

Insight into the mechanisms and kinetics of the solution-mediated phase transformation of theophylline has been provided, by studying the dissolution kinetics of the anhydrous form as well as the crystallization kinetics of the monohydrate 
TABLE 1

Crystallographic features of theophylline

\begin{tabular}{lll}
\hline & Monohydrate $^{\mathrm{a}}$ & Anhydrous $^{\mathrm{b}}$ \\
\hline System & monoclinic & orthorhombic \\
Space group & $\mathrm{P} 2_{1}$ & $\mathrm{Pn}_{2}$ \\
$a(\AA)$ & 13.3 & 24.6 \\
$b(\AA)$ & 4.5 & 3.8 \\
$c(\AA)$ & 15.3 & 8.5 \\
$\beta$ & 99.5 & 90.0 \\
$Z$ & 4 & 4 \\
\hline
\end{tabular}

a Sutor (1958).

b Naqui and Bhattacharyya (1981).

phase. One of our objectives was to explain the behavior of the system by taking into account the above-mentioned processes. Since the change in concentration of theophylline in solution accounts for both crystallization and dissolution, its assessment was chosen as a representative parameter to describe the kinetics of the system. The question is, how does the phase-transformation kinetics lead to the measured theophylline concentration profile in solution?

This system can be described as an isothermal, triphasic, closed system, comprised of two solid phases, anhydrous and monohydrate theophyllinc, and onc liquid phase, theophylline in solution (the different forms in which theophylline can exist in solution need not be considered for the following treatment). As described in Materials and Methods, the system, which is agitated at constant angular velocity, is adjusted to have an initial concentration of theophylline sufficient to achieve the solubility of the anhydrous form, yielding a metastable solution with respect to the monohydrate form. A given amount of both anhydrous and monohydrate crystals of known size distributions is added, inducing the

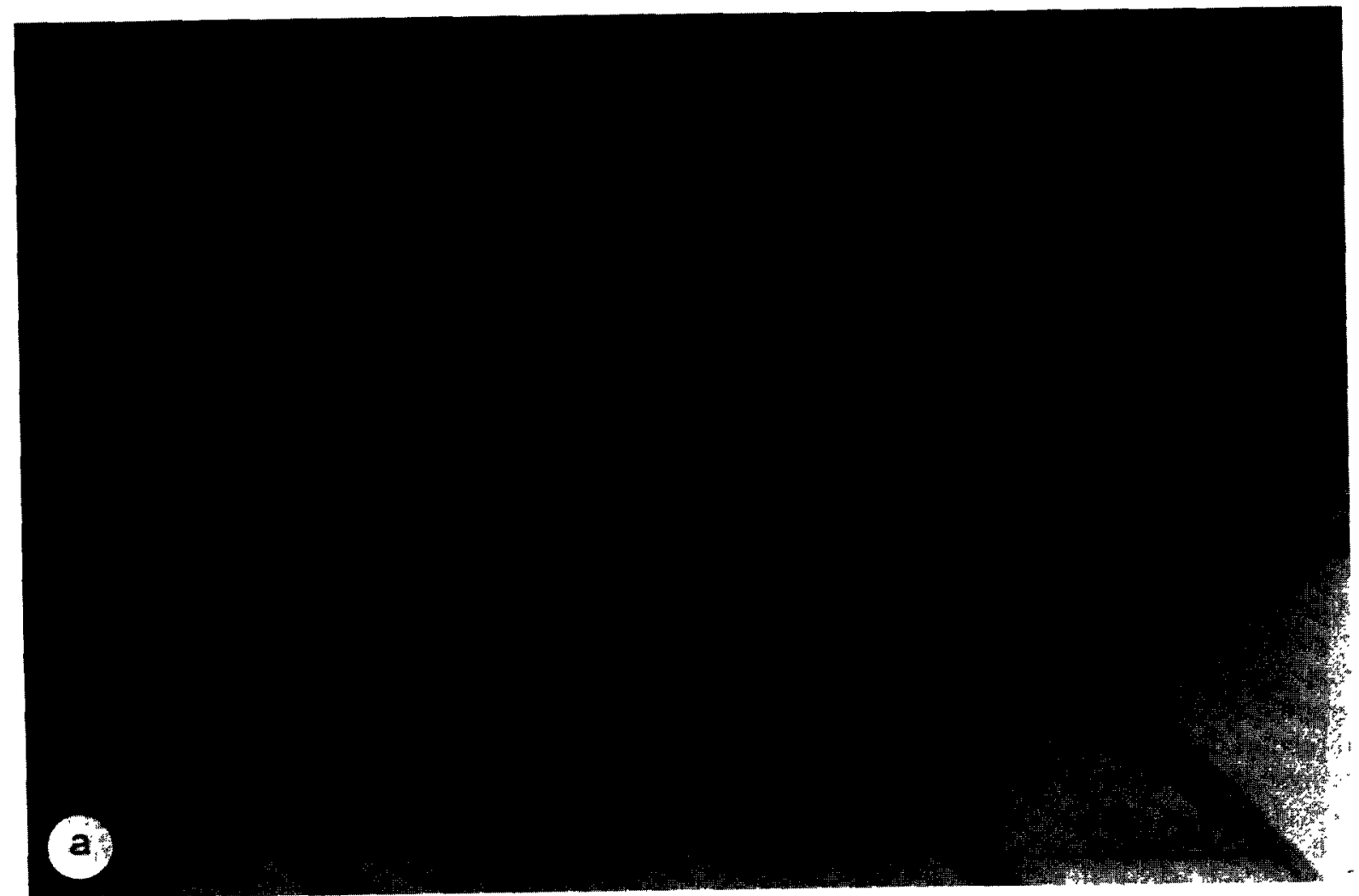

Fig. 9. (a) Theophylline monohydrate, needle-like crystals, growing on the surface of theophylline anhydrous, plate-like single crystal and (b) unit cell of theophylline monohydrate; the fastest growing plane is that along the $b$ crystallographic axis. 

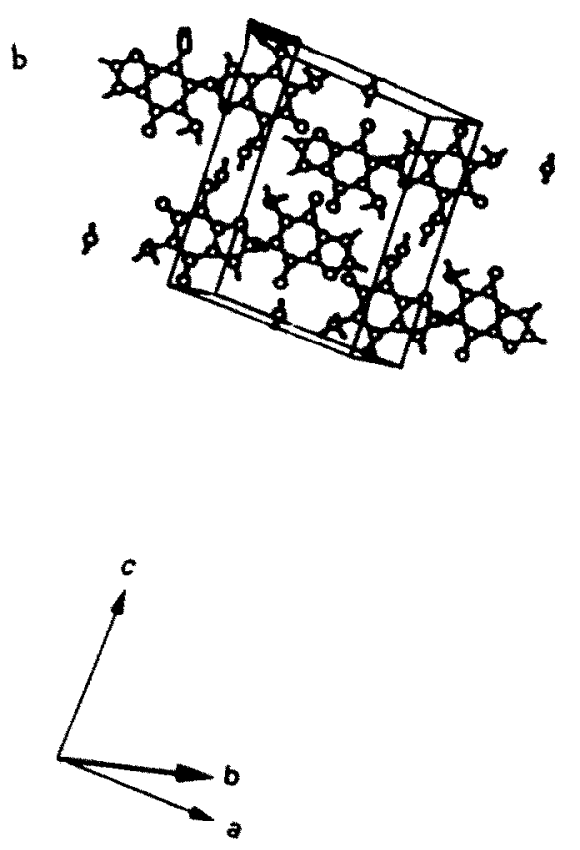

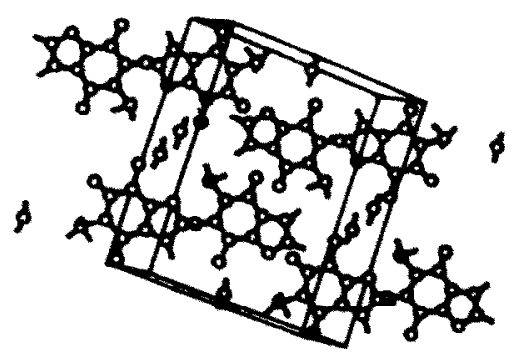

Fig. 9b. formation of monohydrate solid phase and the concomitant dissolution of the anhydrous form. Crystallization of monohydrate theophylline contributes to the depletion of theophylline in solution, due to growth of monohydrate seeds and heterogeneous nucleation and growth of monohydrate crystals. Dissolution of the anhydrous form increases the concentration of theophylline in solution. The observed effect is that the theophylline concentration remains constant as long as the dissolution of the anhydrous solid form keeps up with the precipitation of the monohydrate form. Once all the anhydrous form has dissolved, the growth rate of the monohydrate crystals causes a rapid decrease of theophylline in solution. Finally, the theophylline concentration in solution approaches the solubility of the monohydrate crystals and the transformation is completed.

The value of the instantaneous concentration dictates, in turn, both the degree of supersaturation with respect to monohydrate, $\sigma_{H}$, and the degree of undersaturation with respect to the anhydrous form, $\sigma_{\mathrm{A}}$, driving forces for crystallization and for dissolution, respectively. The kinetic equations that describe the anhydrous phase dissolution (Eqn 4) and the monohydrate phase growth (Eqn 5) are both a function of $\sigma$, but while the growth rate is independent of the crystal size the dissolution rate is not. These rate equations represent average values determined from a given sample of crystals of different sizes. They have been used to simulate the evolution of the CSD during the transformation. The amount and the number of crystals vs size of the monohydrate seeds and the anhydrous crystals are measured at the beginning of an experiment. The CSD on a number basis of the monohydrate nuclei formed during the transformation is evaluated from the final CSD, when the transformation is completed, by taking into account the seeds added initially and the diameter to which they grew during the transformation. The nucleation event is assumed to occur as a burst of monohydrate crystals after the observed lag time in the concentration profile. The narrow spread in size (age) of the measured CSDs supports the validity of this assumption. If nucleation occurred over a large supersaturation range, or time range, the resulting CSD would exhibit a large size 
spread (standard deviation), since crystal growth is not size dependent.

A computer program was written in FORTRAN 77, according to the above description of the system, following the scheme:

(1) Input the initial theophylline concentration in solution, $C_{0}$, and the initial crystal size distribution per unit volume given by the number density functions, $n_{0}\left(L_{i}\right)$, for the anhydrous, monohydrate seeds and monohydrate nuclei.

(2) Calculate the total mass of the anhydrous sceds, monohydrate seeds and monohydrate nuclei in suspension per unit volume (slurry density, $M$ ), for each of the solid phases:

$M_{\mathrm{A}, \mathrm{H}, \mathrm{N}}=\alpha_{v} \rho \sum n_{0}\left(L_{i}\right) L_{i}^{3} \Delta L$

where $\alpha_{\mathrm{v}}$ is the volume shape factor, $\rho$ denotes the crystal density, and $\Delta L$ represents the size interval.

(3) Calculate and save the values of the concentration of theophylline in solution by means of a mass balance, using the previous result to calculate how much theophylline has been dissolved and how much theophylline has been precipitated, i.e.:

$C(t)=C_{0}+C_{\mathrm{A}}-C_{\mathrm{H}}-C_{\mathrm{N}}$

where $C(t)$ denotes the instantaneous concentration of theophylline in solution, $C_{\mathrm{A}}$ is the concentration of the anhydrous form dissolved in $\Delta t$, calculated from the difference of the initial amount of the anhydrous seeds minus the amount of the anhydrous secds from step $2, M_{\mathrm{A}} ; C_{\mathrm{H}}+C_{\mathrm{N}}$ corresponds to the concentration of theophylline depleted by crystallization and is also obtained by difference of the amount of monohydrate calculated in step 2, $M_{\mathrm{H}}$, minus the initial amount of monohydrate in suspension plus the amount of monohydrate formed due to nuclei growth, $M_{\mathrm{N}}$.

(4) Calculate the magnitude of the driving force for dissolution, $\sigma_{\mathrm{A}}$, and for crystallization, $\sigma_{\mathrm{H}}$, from Eqns 1 and 2.

(5) Calculate the instantaneous growth rate:

$G(t)=k_{\mathrm{g}} \sigma_{\mathrm{H}}^{n}$

where $k_{\mathrm{g}}=5.5$ and $n=2.2$.

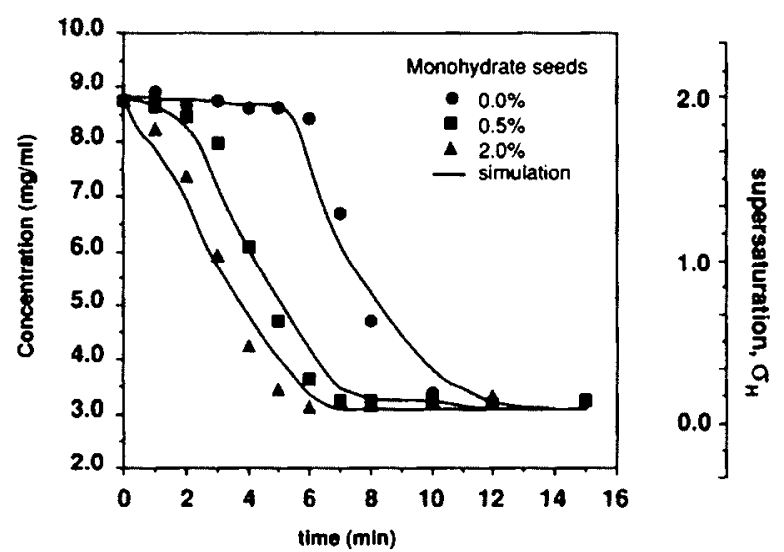

Fig. 10. Observed and predicted concentration profiles.

(6) Calculate the instantaneous dissolution rate:

$D(t)_{i}=k_{\mathrm{d}} \sigma_{\mathrm{A}}^{m} L_{i}^{b}$

where $k_{\mathrm{d}}=500, b=-0.5$, and $m=1.5$.

(7) Calculate new crystal sizes:

$L_{i}=L_{i-1}-D(t)_{i-1} \Delta t \quad$ (anhydrous form)

$L_{i}=L_{i-1}+G(t) \Delta t \quad$ (monohydrate)

(8) Assign the new sizes to the old values in the number density function to obtain the new size distributions for all the solid phases: anhydrous seeds, monohydrate seeds and monohydrate nuclei:

$n\left(L_{i-1}\right)=n\left(L_{i}\right)$

(9) Repeat steps 2-8 and stop when $L_{i}=0$ for the anhydrous form and $L_{i}=L_{i-1}$ for the monohydrate solid phase.

The results of the simulation are shown in Fig. 10.

\section{Conclusions}

The results of the present study show that the anhydrous to hydrate transformation of theo- 
phylline occurs by a solvent-mediated process. Insight into the mechanisms responsible for the transformation is provided, by measuring the concentration profile and the evolution of the CSD during the phase transformation. By independently studying the processes involved, dissolution and crystallization, the transformation kinetics has been described.

Anhydrous theophylline crystals act as heterogeneous nucleation substrates for the crystals of the monohydrate form and the new phase grows epitaxially on the anhydrous crystals. This has significant consequences on the choice of a metastable or a stable solid phase of a drug in a formulation, since it is frequently assumed that the metastable phase would dissolve faster. However, if the metastable phase is a heterogeneous nucleant for the stable phase, the dissolution of the metastable phase will be inhibited, and the anticipated drug concentration levels will not be achieved. This explains the observed instability of drug release from anhydrous theophylline dosage forms.

\section{Acknowledgements}

This work was supported in part by a Biomedical Research Support Grant at the University of Arizona, and by a Young Investigator Award from Eli Lilly.

\section{References}

Amathieu, L. and Boistelle, R., Crystallization kinetics of gypsum from dense suspension of hemihydrate in water. $J$. Crystal Growth, 88 (1988) 183-192.

Ando, H., Ohwaki, T., Ishii, M., Watanabe, S. and Miyake, Y., Crystallization of theophylline in tablets. Int. J. Pharm., 34 (1986) 153-156.

Boistelle, R. and Rinaudo, C., Phase transition and epitaxies between hydrated orthorhombic and anhydrous monoclinic uric acid crystals. J. Crystal Growth, 53 (1981) 1-9.

Burton, W.K., Cabrera, N. and Frank, F.C., The growth of crystals and the equilibrium structures of their surfaces. Phil. Trans. Roy. Soc., A243 (1951) 299-358.

De Smidt, J.G., Fokkens, J.G., Grijseels, H. and Crommelin, D.J.A., Dissolution of theophylline monohydrate and anhydrous theophylline in buffer solutions. J. Pharm. Sci., 75 (1986) 497-501.

\section{Glossary}

\begin{tabular}{|c|c|}
\hline Symbol & Meaning \\
\hline$(\mathrm{d} C / \mathrm{d} t)_{0}$ & initial dissolution rate \\
\hline$C$ & concentration in solution \\
\hline$C(t)$ & instantaneous concentration \\
\hline$D$ & dissolution rate \\
\hline$D(t)$ & instantaneous dissolution rate \\
\hline$G$ & growth rate \\
\hline$G(t)$ & instantaneous growth rate \\
\hline$k_{\mathrm{d}}$ & dissolution rate constant \\
\hline$k_{\mathrm{g}}$ & growth rate constant \\
\hline$L$ & crystal size \\
\hline$M$ & slurry density \\
\hline$n(L)$ & number density function \\
\hline$S$ & solubility \\
\hline$\alpha_{v}$ & shape factor \\
\hline$\Delta \mu_{\mathrm{D}}$ & $\begin{array}{l}\text { change in chemical potential for dissolution } \\
\text { change in chemical potential for crystallization }\end{array}$ \\
\hline$\rho$ & crystal density \\
\hline$\sigma$ & $\begin{array}{l}\text { driving force: supersaturation (crystallization); } \\
\text { undersaturation (dissolution) }\end{array}$ \\
\hline \multicolumn{2}{|l|}{ Subscripts } \\
\hline A & anhydrous \\
\hline $\mathbf{H}$ & monohydrate \\
\hline$i$ & iteration number \\
\hline $\mathrm{N}$ & nuclei \\
\hline \multicolumn{2}{|l|}{ Superscripts } \\
\hline$m$ & reaction order \\
\hline$n$ & growth exponent \\
\hline
\end{tabular}

Fokkens, J.G., Van Amelsfoort, J.G.M., De Blaey, C.J., De Kruif, C.G. and Wilting, J., A thermodynamic study of the solubility of theophylline and its hydrate. Int. J. Pharm., 14 (1983) $79-93$.

Herman, J,, Remon, J.P., Visavarungroj, N., Schwartz, J.B. and Klinger, G.H., Formation of theophylline monohydrate during the pelletisation of microcrystalline cellulose-anhydrous theophylline blends. Int. J. Pharm., 42 (1988) $15-18$

Herman, J., Visavarungroj, N. and Remon, J.P., Instability of drug release from anhydrous theophylline-microcrystalline cellulose formulations. Int. J. Pharm., 55 (1989) 143-146.

Naqvi, A.A., and Bhattacharyya, G.C., Crystal data for anhydrous theophylline. J. Appl. Crystallogr., 14 (1981) 464.

Rodríguez-Hornedo, N. and Wu, H.J., Crystal growth kinetics of theophylline monohydrate. Pham. Res., 8 (1991) 643648 .

Shefter, E. and Higuchi, T, Dissolution behavior of crystalline solvated and nonsolvated forms of some pharmaceuticals. J. Pharm. Sci., 52 (1963) 781-791. 
Sutor, J., The structure of pyrimidines and purines. VI. The crystal structure of theophylline. Acta Crystallogr., 11 (1958) 83-87.

Wadke, D.A. and Reier, G.E., Use of intrinsic dissolution rates to determine thermodynamics parameters associated with phase transitions. J. Pharm. Sci., 61 (1972) 868-871.

Walton, A.G., Nucleation in liquids and solutions. In Zettle- moyer, A.C. (Ed.), Nucleation. Dekker, New York, 1969, p. 273.

Windholz, M. (Ed.), The Merck Index, 10th Edn, Merck, Rahway, NJ, 1983, p. 1328.

$\mathrm{Wu}, \mathrm{H} . J .$, The kinetics of solvent mediated phase transformations, Ph.D. Thesis, University of Arizona, Tucson, 1990. 